\title{
Second International Workshop on Gamification for Information Retrieval (GamifIR'15)
}

\author{
Frank Hopfgartner ${ }^{1}$, Gabriella Kazai ${ }^{2}$, Udo Kruschwitz ${ }^{3}$, Michael Meder ${ }^{4}$, and \\ Mark Shovman ${ }^{5}$ \\ 1 University of Glasgow, UK \\ 2 Semion Ltd., UK \\ 3 University of Essex, UK \\ 4 Technische Universität Berlin, Germany \\ 5 Yahoo! Labs, Israel
}

\begin{abstract}
Gamification is a popular methodology describing the trend of applying game design principles and elements, such as feedback loops, points, badges or leader boards in non-gaming environments. Gamification can have several different objectives. Besides just increasing the fun factor, these could be, for example, to achieve more accurate work, better retention rates and more cost effective solutions by relating motivations for participating as more intrinsic than conventional methods. In the context of Information Retrieval (IR), there are various tasks that can benefit from gamification techniques such as the manual annotation of documents in IR evaluation or participation in user studies to tackle interactive IR challenges. Gamification, however, comes with its own challenges and its adoption in IR is still in its infancy. Given the enormous response to the first GamifIR workshop at ECIR 2014 and the broad range of topics discussed it seemed timely and appropriate to organise a follow-up workshop.
\end{abstract}

\section{Background and Motivation}

Many research challenges in the field of IR rely on tedious manual labour. For example, manual feedback is required to assess the relevance of documents to a given search task, to annotate documents or to evaluate interactive IR approaches. A recent trend to perform these tasks is the use of crowdsourcing techniques, i.e., obtaining relevance labels from anonymous crowd workers via an open call. Although research indicates that such techniques can be useful, they fail when motivated users are required to perform a task for reasons other than just being paid per click, document judged or time spent on the task.

A promising approach to increase user motivation is by employing gamification methods which has been applied in various environments and for different purposes such as marketing, education, pervasive health care, enterprise workplaces, e-commerce, human resource management and many more. The definition of gamification is still under discussion, e.g., whether it covers methods "to facilitate and support the users' overall value creation"[?] or as a user experience enhancement using game design elements "regardless of specific usage 
intentions, contexts [...]"[?] or environments. Definitions pursuing the increase of user experience and overall value indicate that the application of gamification is goal-oriented. Although several studies indicate that gamification can lead to increased user activity, a detailed analysis of users' personal perception of gamification principles has barely been studied. In the last few years, several frameworks on how to 'gamify' were proposed, but there are still many open questions on how to start. We think a particular challenge of applying gamification is to find an elegant and subtle way of adopting and adapting game design patterns, mechanisms and elements to a particular problem or scenario.

The purpose of the GamifIR workshops is to bring together researchers and practitioners from a wide range of areas including game design, IR, humancomputer interaction, computer games, and natural language processing in order to start a discussion and an exchange of research ideas and results relating to emerging areas of gamification within the context of IR.

The First International Workshop on Gamification in Information Retrieval (GamifIR'14) was held at ECIR 2014 in Amsterdam (half day only). The workshop focused on the challenges and opportunities that gamification may present for the information retrieval (IR) community [1]. ${ }^{6}$ In response to the call for papers, 18 submissions were received, out of which 14 were accepted for presentation at the workshop. Over 40 people attended the workshop, representing both industry and academia. Given the interest of the first GamifIR workshop created in the run-up of the event and the discussions emerging at the workshop, we are convinced that we are only at the start of seeing gamification becoming an established methodology to support and push forward IR in a variety of ways. This - we believe - merits the organisation of a second GamifIR workshop.

\section{Workshop Goals}

The call for papers solicited submissions of position papers as well as novel research papers and demos addressing problems related to gamification and IR including topics such as:

- Gamification approaches in a variety of contexts, including document annotation and ground-truth generation; interface design; information seeking; user modelling; knowledge sharing

- Gamification design

- Applied game principles, elements and mechanics

- Gamification analytics

- Long-term engagement

- User engagement and motivational factors of gamification

- Player types, contests, cooperative gamification

- Search challenges and gamification

\footnotetext{
${ }^{6}$ A detailed review of the workshop can be found in the Spring 2014 edition of Informer, the quarterly newsletter of the BCS IRSG at http://irsg.bcs.org/ informer/2014/04/gamifir-2014/
} 
- Game based work and crowdsourcing

- Applications and prototypes

Submissions from outside the core IR community and from industry were actively encouraged.

Detailed information about the workshop can be found on the workshop website at http://gamifir.dai-labor.de/organizers/.

\section{Keynote}

We were very pleased that Dr Leif Azzopardi of the University of Glasgow could be convinced to give a keynote talk at GamifIR'15. Leif is a well-known IR character who bridges different research communities and has a particular interest in user interactions. His work fits in very nicely with the overall aims of the workshop.

\section{Acknowledgements}

We acknowledge the efforst of the programme committee, namely:

- Omar Alonso, Microsoft Research (USA)

- Raian Ali, Bournemouth University (UK)

- Michael Ameling, SAP (Germany)

- Jon Chamberlain, University of Essex (United Kingdom)

- Carsten Eickhoff, ETH Zurich (Switzerland)

- Christopher G Harris, The University Of Iowa (USA)

- Hideo Joho, University of Tsukuba (Japan)

- Edith Law, University of Waterloo (Canada)

- Till Plumbaum, TU Berlin (Germany)

- Craig Stewart, Coventry University (United Kingdom)

- Albert Weichselbraun, University of Applied Sciences Chur (Switzerland)

\section{References}

1. F. Hopfgartner, G. Kazai, U. Kruschwitz, and M. Meder, editors. GamifIR '14: Proceedings of the First International Workshop on Gamification for Information Retrieval, New York, NY, USA, 2014. ACM. 